\title{
Hidden in Plain Sight: Enhanced Decision Making in an Unstable Patient
}

\author{
Nikos Pappan ${ }^{1}$, Andreas Kyvernitakis ${ }^{1}$, Tarun Tewatia ${ }^{2}$, Rachel Hughes-Doichev ${ }^{2}$, \\ Kartikeya Kashyap ${ }^{1}$, Craig Alpert ${ }^{2}$, and Erica Marshall ${ }^{2}$ \\ ${ }^{1}$ Affiliation not available \\ ${ }^{2}$ Allegheny General Hospital
}

April 29, 2020

\begin{abstract}
The use of ultrasound enhancing agents (UEA) help optimize visualization in technically difficult studies, with improved left ventricular opacification and endocardial border definition. The use of these agents may often unveil critical data that drastically alter clinical management. Despite the clinical benefit of using UEA, it is sometimes not used routinely in emergency situations with an unstable patient for many reasons. Herein, we demonstrate a challenging case of late presentation myocardial infarction complicated with cardiogenic shock and pseudoaneurysm formation that was not observed in non-contrast images emblematically demonstrating how the use of ultrasound enhancing agents can drastically impact clinical decision-making.
\end{abstract}

\section{Background}

The use of ultrasound enhancing agents (UEA) in echocardiography is a technique that can be used to enhance endocardial border delineation in studies with suboptimal left ventricular cavity visualization [1-3]. There are two different types of contrast agents that serve different purposes. The first one is agitated saline contrast which is frequently utilized to identify small right-to-left shunts such as patent foramen ovale or atrial septal defect [4]. The other is a newer technique consisting of microbubble contrast agents which traverse the pulmonary vasculature and opacify the left ventricle (LV), allowing for accurate endocardial border definition in patients with suboptimal echocardiograms [1]. This allows for improved sensitivities and specificities when comparing patients with poor echocardiographic windows to those with well visualized windows $[3,5]$. In a large prospective study, patients with technically difficult echocardiographic studies were evaluated to see how UEA effected diagnosis and management. The percentage of uninterpretable studies decreased from $11.7 \%$ to $0.3 \%$. Moreover, further procedures were avoided in $33 \%$ of patients and medical management was altered in 10\% [1]. By decreasing need for additional procedures, use of UEA is cost effective. In terms of safety profile with UEAs, back pain is the most common complaint followed by headache and allergic reactions [6]. Application of UEAs have not been associated with significant extra cost of time and manipulations because small bolus injections have been shown to suffice for image quality [7]. Indeed, they have been shown to be cost-effective (4) and life-saving [8].

\section{Case report}

An 87 year-old woman with hypertension, diabetes, coronary artery disease, and an ischemic cardiomyopathy presented with acute coronary syndrome and cardiogenic shock. Prior to abrupt clinical deterioration, she had described angina and heart failure symptoms of two weeks' duration. On presentation, she was hypotensive requiring vasopressor support along with laboratory evidence of multiorgan failure. Electrocardiography demonstrated biventricular paced rhythm with a lateral wall infarct. 
She was admitted to the cardiac intensive care unit and a bedside transthoracic echocardiogram revealed apical and lateral wall akinesis as well as aortic valve thickening. Due to suboptimal visualization of the LV and anticipated need for mechanical circulatory support, UEA was administered to exclude LV thrombus prior to proceeding with coronary angiography. The bedside echocardiogram revealed possible pericardial effusion versus a prominent pericardial fat; however, with the use of UEA, a large, expansive lateral wall pseudoaneurysm with a narrow neck width of $1.1 \mathrm{~cm}$ was revealed (Figure 1). UEA also opacified the pericardial space, revealing a large pericardial effusion with extensive thrombus.

\section{Discussion}

Currently, Cardiology societies recommend the use of UEA when $>2$ contiguous LV segments are not visible on non-contrast images [9]. Herein, we demonstrate a case where routine beside echocardiography was limited by poor visualization of the LV endocardial borders highlighting the critical impact UEA may have on clinical decision making.

In our patient, the initial treatment plan was for emergent percutaneous coronary revascularization and temporary left ventricular assist device placement. However, the use of UEA with a focused study completely transformed patient management. UEA allowed us to identify a large LV pseudoaneurysm which would have rendered any such interventions potentially catastrophic. Instead, we consulted cardiothoracic surgery emergently to evaluate for open heart surgical revascularization and repair of the LV pseudoaneurysm. The use of echocardiography with UEA in this patient was potentially life-saving and prevented a potentially disastrous iatrogenic complication.

\section{Conclusion}

We present a case where the use of UEA in an acutely unstable patient completely altered clinical medical decision making by revealing a large LV pseudoaneurysm that was not visualized by non-contrast echocardiography. In emblematic occasions such as this, UEA can be life-saving revealing previously occult yet ominous pathophysiology, especially in a patient in cardiogenic shock.

\section{Author Contributions}

Nikos Pappan and Andreas Kyvernitakis drafting and revising original manuscript. Kartik Kashyap, Erica Marshall, Craig M. Alpert, Robert W.W. Biederman, and Rachel A. Hughes-Doichev final revisions and approval of final manuscript.

\section{References}

1. Kurt M, Shaikh KA, Peterson L et al. Impact of contrast echocardiography on evaluation of ventricular function and clinical management in a large prospective cohort. J Am Coll Cardiol 2009;53(9):802-810.

2. Hundley WG, Kizilbash AM, Afridi I, Franco F, Peshock RM, Grayburn PA. Administration of an intravenous perfluorocarbon contrast agent improves echocardiographic determination of left ventricular volumes and ejection fraction: comparison with cine magnetic resonance imaging. J Am Coll Cardiol 1998;32(5):14261432 .

3. Nanda NC, Kitzman DW, Dittrich HC, Hall G, Imagent Clinical Investigators G. Imagent improves endocardial border delineation, inter-reader agreement, and the accuracy of segmental wall motion assessment. Echocardiography 2003;20(2):151-161.

4. Attaran RR, Ata I, Kudithipudi V, Foster L, Sorrell VL. Protocol for optimal detection and exclusion of a patent foramen ovale using transthoracic echocardiography with agitated saline microbubbles. Echocardiography 2006;23(7):616-622.

5. Dolan MS, Riad K, El-Shafei A et al. Effect of intravenous contrast for left ventricular opacification and border definition on sensitivity and specificity of dobutamine stress echocardiography compared with coronary angiography in technically difficult patients. Am Heart J 2001;142(5):908-915. 
6. Kumar S, Purtell C, Peterson A, Gibbons P, Khan AM, Heitner SB. Safety profile of ultrasound enhancing agents in echocardiography. Echocardiography 2019;36(6):1041-1044.

7. Jung PH, Angermann CE. Clinical benefits of using second generation ultrasound contrast agents in stress echocardiography. Echocardiography 2003;20 Suppl 1:S11-18.

8. Kusnetzky LL, Khalid A, Khumri TM, Moe TG, Jones PG, Main ML. Acute mortality in hospitalized patients undergoing echocardiography with and without an ultrasound contrast agent: results in 18,671 consecutive studies. J Am Coll Cardiol 2008;51(17):1704-1706.

9. American College of Cardiology Foundation Appropriate Use Criteria Task F, American Society of E, American Heart A et al. ACCF/ASE/AHA/ASNC/HFSA/HRS/SCAI/SCCM/SCCT/SCMR 2011 Appropriate Use Criteria for Echocardiography. A Report of the American College of Cardiology Foundation Appropriate Use Criteria Task Force, American Society of Echocardiography, American Heart Association, American Society of Nuclear Cardiology, Heart Failure Society of America, Heart Rhythm Society, Society for Cardiovascular Angiography and Interventions, Society of Critical Care Medicine, Society of Cardiovascular Computed Tomography, and Society for Cardiovascular Magnetic Resonance Endorsed by the American College of Chest Physicians. J Am Coll Cardiol 2011;57(9):1126-1166.

\section{Figure Header}

\section{Figure 1: Echocardiography Imaging with and without an Ultrasound Enhancing Agent.}

A. Apical 4-chamber view showing suboptimal endocardial visualization. B. Apical 4-chamber view following the use of intravenous contrast enhancement showing extravasated contrast into the pericardial space revealing the pseudoaneurysm (arrow). C.Parasternal short-axis view with poor endocardial border definition.D. Parasternal short-axis view at the same level revealing a rupture in the left ventricle wall and communication with the pericardial space revealing the pseudoaneurysm (arrow).

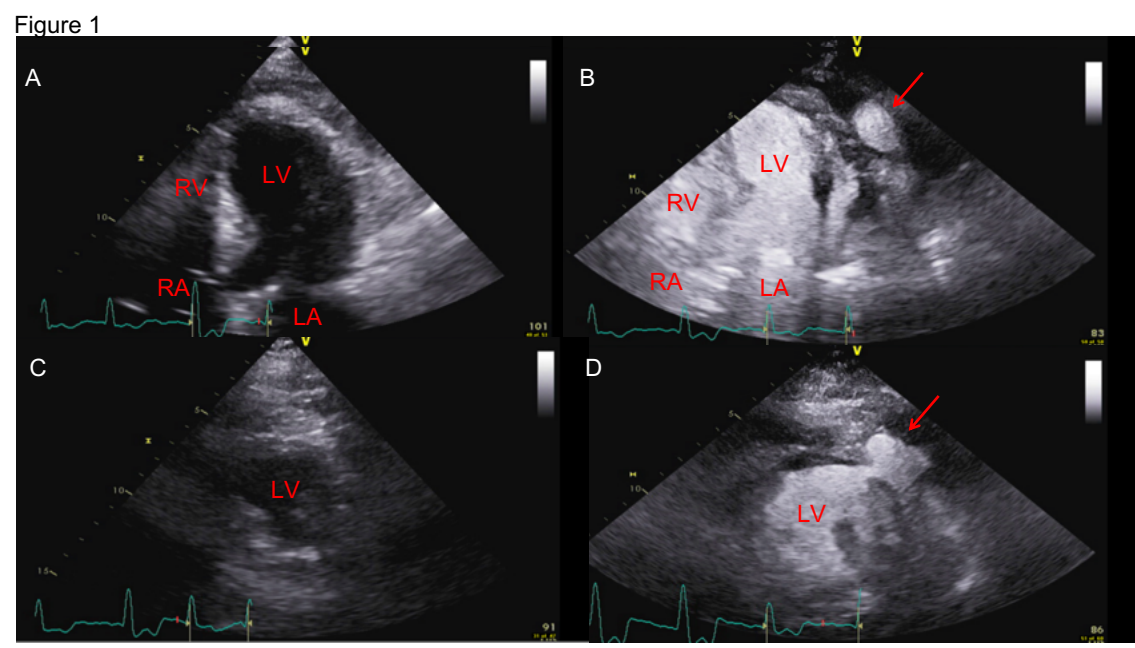

$\mathrm{LV}=$ Left Ventricle, $\mathrm{RV}=$ Right Ventricle, $\mathrm{LA}=$ Left Ventricle, $\mathrm{RA}=$ Right Ventricle, Arrow=Pseudoaneurysm 